\title{
Derivation of parameters used in Spectrum for eligibility for antiretroviral therapy and survival on antiretroviral therapy
}

\author{
Mary Mahy, ${ }^{1}$ Charlotte Lewden, ${ }^{2}$ Martin W G Brinkhof, ${ }^{3}$ Francois Dabis, ${ }^{2}$ \\ Jean-Michel Tassie, ${ }^{4}$ Yves Souteyrand, ${ }^{4}$ John Stover $^{5}$
}

\begin{abstract}
1 UNAIDS, Geneva, Switzerland ${ }^{2}$ Université Victor Segalen Bordeaux 2, Institut de Santé Publique d'Epidémiologie et de Developpement (ISPED), Bordeaux, France, INSERM, U597, Bordeaux, France ${ }^{3}$ Swiss Paraplegic Research, Nottwil, Switzerland

${ }^{4}$ World Health Organization, Geneva, Switzerland

${ }^{5}$ Futures Institute, Glastonbury, Connecticut, USA
\end{abstract}

\section{Correspondence to}

Dr Mary Mahy, UNAIDS, 20

Avenue Appia, 1211 Geneva 27,

Switzerland;

mahym@unaids.org

Accepted 27 September 2010

\section{ABSTRACT}

Background The Spectrum projection package uses estimates of national HIV incidence, demographic data and other assumptions to describe the consequences of the HIV epidemic in low and middle-income countries. The default parameters used in Spectrum are updated every 2 years as new evidence becomes available to inform the model. This paper reviews the default parameters that define the course of HIV progression among adults and children in Spectrum.

Methods For adults, data available from published and grey literature and data from the ART-LINC International epidemiologic Database to Evaluate AIDS (leDEA) collaboration were combined to estimate survival among those who started antiretroviral therapy (ART). For children, a review of published material on survival on ART and survival on ART and cotrimoxazole was used to derive survival probabilities. Historical data on the distribution of CD4 cell counts and CD4 cell percentages by age among children who were not treated (before treatment was available) were used to progress children from seroconversion to different CD4 cell levels.

Results Based on the updated evidence estimated survival among adults aged over 15 years in the first year on ART was $86 \%$, while in subsequent years survival was estimated at $90 \%$. Survival among children during the first year on ART was estimated to be $85 \%$ and for subsequent years $93 \%$.

Discussion The revised default parameters based on additional data will make Spectrum estimates more accurate than previous rounds of estimates.

Every 2 years UNAIDS and partners support low and middle-income countries to create national estimates and projections of their HIV epidemics with modelling software. The estimation and projection package (EPP) is used to create a national HIV incidence curve. ${ }^{1}$ The Spectrum computer package then uses the HIV incidence curve to project the impact. $^{2}$

To make accurate estimates and projections countries must populate EPP and Spectrum with HIV sentinel surveillance data, HIV prevalence from population-based surveys (if available) and programme data such as the number of women receiving antiretroviral prophylaxis to prevent mother-to-child transmission of HIV and the number of adults receiving antiretroviral therapy (ART). In addition, EPP and Spectrum use numerous parameters that determine how HIV progresses in a given individual and how it affects the demographic structure of the population. Of specific importance for estimating ART needs and its impact are parameters that describe the time from HIV seroconversion until the need for treatment and the time from treatment until death. If countries have local studies that capture these parameters, the country-specific data can be used in the model, reflecting the situation in their country. If no data are available from the country on the specific parameter default parameters are used in the model.

Outputs of Spectrum include the number of people living with HIV, estimated HIV prevalence, number of deaths due to AIDS, number of people in need of ART, number of HIV-positive pregnant women in need of antiretroviral prophylaxis to avoid transmission to children, and the estimated number of orphans due to AIDS. The Spectrum outputs are used by countries to develop their national strategic plans, to identify and set treatment targets, and to estimate the impact of ART and prevention of mother-to-child transmission programmes at the population level. ${ }^{3-5}$ The models thus need to be as accurate as possible and should precisely reflect the current level of the epidemic in the specific country.

As more data become available from ART programmes and cohort studies, the default parameters used in Spectrum are updated and improved. Here we describe how the default parameters related to HIV progression were determined for the version of Spectrum used in the 2009 round of national HIV estimates. In particular, we explain the derivation of parameters on: seroconversion to eligibility for treatment for adults and children; survival among those starting on ART in the first year on ART and in subsequent years, for adults and children.

Spectrum is a compartmental model covering three epidemic states (HIV negative, HIV positive and not in need of treatment, and HIV positive and in need of treatment) and death. It is a discrete model based on age groups. The population data used in Spectrum come from the UN Population Division's population prospects 2008 revision. More information on Spectrum is available elsewhere. ${ }^{2} 6$

\section{ADULTS}

In Spectrum survival time from seroconversion to death is split into two periods: time from seroconversion to eligibility for treatment and time from eligibility for treatment until death. The 
patterns describing overall progression from new seroconversion to AIDS death without treatment have not changed since the 2008 round of estimates and are described elsewhere. ${ }^{78}$ In brief, the progression patterns are based on demographic surveillance sites in the ALPHA network in the absence of ART. ${ }^{9}$

\section{Progression from seroconversion to eligibility for treatment}

As early as 2005 low and middle-income countries started changing national guidelines to initiate individuals on ART earlier in the course of HIV disease progression. To reflect these changes, Spectrum now includes an option allowing countries to change adult eligibility criteria for ART in the model. The version of Spectrum used in 2009 assumes that eligibility was defined initially as all HIV-infected adults with a CD4 cell count under 200 cells $/ \mathrm{mm}^{3}$. If eligibility criteria have changed they can be defined as CD4 cell counts under 250 or 350 cells $/ \mathrm{mm}^{3}$ and the year the change was implemented. The time from seroconversion until the need for treatment is adjusted based on the eligibility criterion.

The previous version of Spectrum in 2007 used data from the eligibility for ART in lower income countries collaboration (eART-linc) to determine time from seroconversion to eligibility for treatment (assuming eligibility was $200 \mathrm{cells} / \mathrm{mm}^{3}$ ). The eART-linc collaboration compiled data from five treatment programmes in low and middle-income countries to measure the evolution of CD4 cell counts over the progression of the disease.
The five sites included in the eART-linc data include two sites in Uganda, two sites in Thailand and one site in Côte d'Ivoire. ${ }^{10}$

In the 2009 version of Spectrum the eART-linc results were again used to describe the time from seroconversion to eligibility and from eligibility to death using three different eligibility criteria: CD4 less than 200 cells/mm $\mathrm{mm}^{3}$, CD4 less than 250 cells/ $\mathrm{mm}^{3}$ and CD4 less than 350 cells $/ \mathrm{mm}^{3}$. The progression patterns were developed by fitting Weibull curves to match the median progression time to eligibility and to death and matching the overall pattern of progression from seroconversion to death as estimated by the ALPHA network cohort studies. ${ }^{8}$ The Weibull patterns are implemented in Spectrum by converting them to annual survival rates as a function of time since infection.

Two patterns of progression were included in Spectrum and were classified as slow and fast progression. The fast progression was based on data from a Thai military cohort, which showed more rapid progression through HIV stages to death (median progression times are 8.1 years for men and 8.9 years for women). The fast progression pattern is used by a few countries in Asia with evidence of similar patterns, whereas the vast majority of countries use the slow progression pattern. Figure 1 shows the resulting estimates used for Spectrum for the 200 and 350 cells $/ \mathrm{mm}^{3} \mathrm{CD} 4$ cell threshold values assuming no treatment for the slow progression pattern. For men the median time from seroconversion to eligibility was reduced from 8.2 to 5.8 years
Figure 1 HIV progression by CD4 cell eligibility criterion using the slow pattern for males and females.
Eligibility at $\mathrm{CD} 4<200 \mathrm{~mm} 3$ : Males

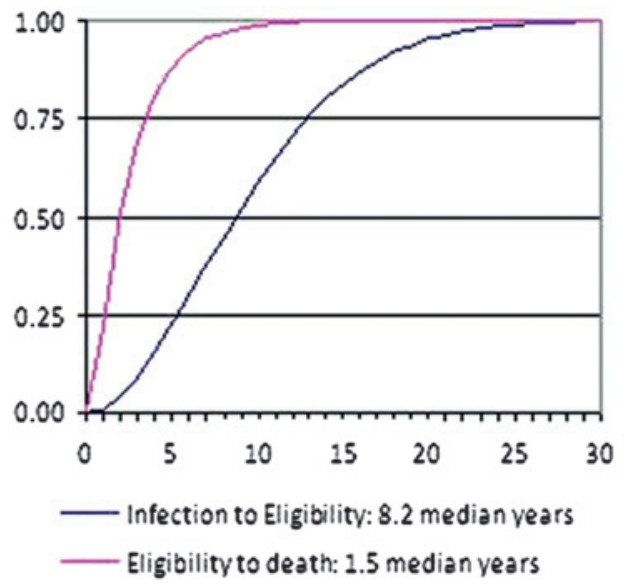

Eligibility at CD $4<350 \mathrm{~mm} 3$ : Males

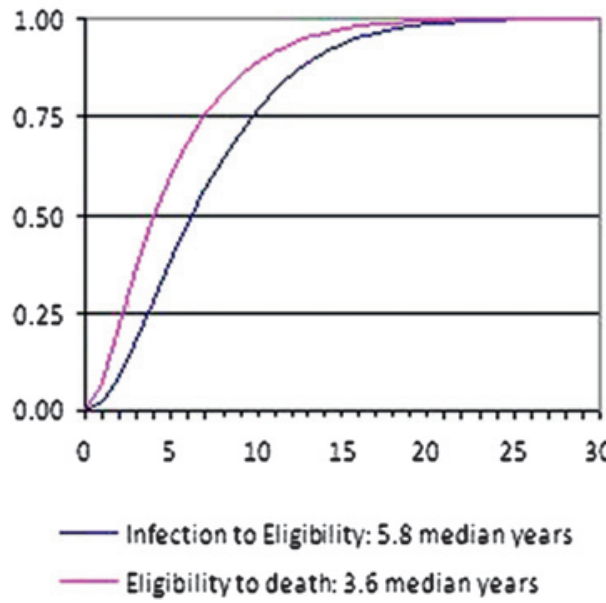

Eligibility at $\mathrm{CD} 4<200 \mathrm{~mm} 3$ : Females

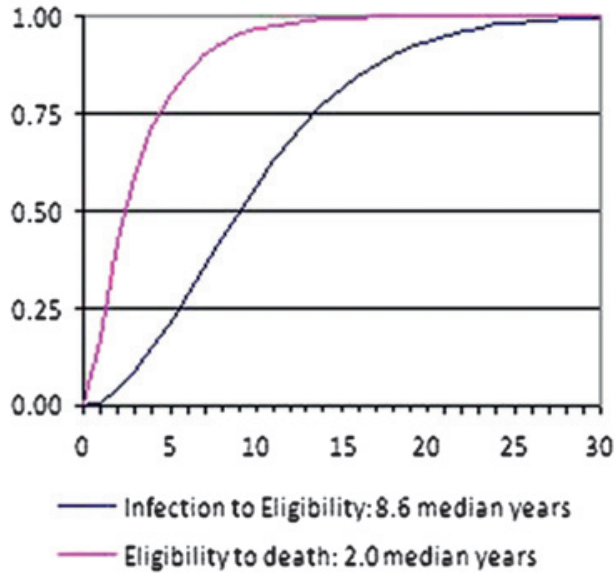

Eligibility at $C D 4<350 \mathrm{~mm} 3$ : Females

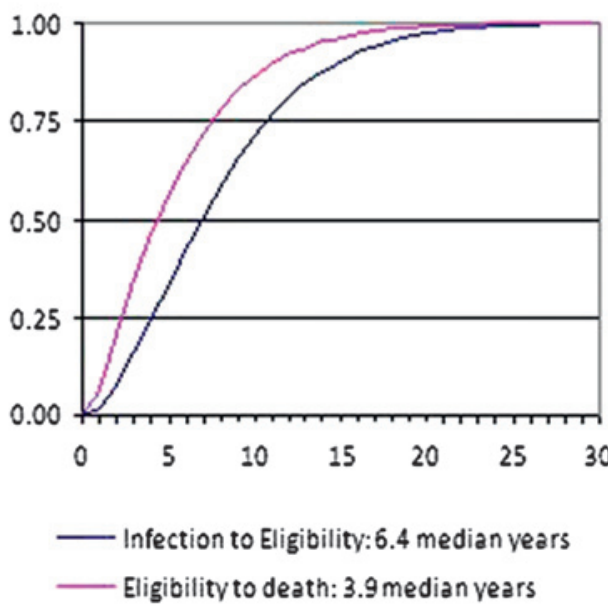


when switching ART eligibility criteria from 200 cells $/ \mathrm{mm}^{3}$ to 350 cells $/ \mathrm{mm}^{3}$ (the mean progression time is reduced from 9.1 to 6.7 years). For women the median time from seroconversion to eligibility was reduced from 8.6 to 6.4 years when switching the eligibility criteria from 200 cells $/ \mathrm{mm}^{3}$ to $350 \mathrm{cells} / \mathrm{mm}^{3}$ (the mean progression time is reduced from 9.5 to 7.4 years).

\section{Survival on ART}

To derive the parameter of survival on ART two datasets were reviewed: the first included the review of individual data from a pooled dataset from a collaborative study and the second was a literature review of published or presented studies.

Pooled data were analysed from the ART-LINC of the International epidemiologic Database to Evaluate AIDS (IeDEA) collaboration. This collaboration (not directly related to eARTlinc described above) was established in 2003 to characterise the prognosis of HIV-infected patients treated with ART in resource-limited settings, to compare the experience between different settings, delivery modes and types of monitoring, and to compare outcomes with those observed in industrialised nations. The ART-LINC collaboration was composed of 19 cohort studies and two networks. The data came from the following countries: Uganda, Rwanda, Burundi, Kenya, Côte d'Ivoire, Senegal, Nigeria, Morocco, Zimbabwe, Zambia, South Africa, Botswana, Malawi, Congo, DR Congo, Thailand, India, Brazil and Argentina. The data submitted from these sites had similar data formats allowing the calculation of pooled indicators and comparisons between sites. ${ }^{11}$ The last data merge for the ART-LINC collaboration occurred in 2007-8. Among the patients in this dataset the baseline median CD4 cell count at the start of ART was 113 cells $/ \mathrm{mm}^{3}$ (IOR 47-187), the median age was 35 years (IOR 30-41), 60\% were women and 54\% were either WHO stages 3 or 4 .

In addition, a literature review covering the period $2001-6^{12}$ and updated to cover 2007 and 2008 was conducted to estimate the survival time on ART. ${ }^{13}$ The reviewers screened PubMed/ Medline and Scopus for the 2001-6 review and additionally searched Current Contents Connect and abstracts from the 2008 international conferences for the 2007 and 2008 update. Eligible reports included original prospective studies in which adults had started ART in low and middle-income countries, with reports of deaths or losses to follow-up. A total of 26 studies was used for the 2001-6 review. An additional 103 articles and 33 abstracts from international conferences were reviewed in the updated analysis.

The parameter for survival on ART was derived primarily from survival analysis of cohorts or programmes in lower and middleincome countries primarily using eligibility criteria for starting ART of WHO stages 3 or 4 or a CD4 cell count less than 200 cells $/ \mathrm{mm}^{3}$. However, evidence suggests that survival will change as countries adjust the criteria to start patients on ART earlier, ${ }^{14}{ }^{15}$ as adopted by the WHO at the end of $2009 .{ }^{16}$ Ideally, the models would describe survival based on the CD4 cell levels at the start of treatment. At the time the Spectrum revisions were made there was very little evidence on which to base an assumption about better survival with an earlier start on ART. In addition, very few countries currently have national data to include in a model from which a graduated survival curve based on starting CD4 cell levels could be calculated.

Analysis of the pooled ART-LINC data from 2008 suggested that the probability of death at 1 year was estimated to be 0.06 (0.064 in Africa, 0.048 in Asia and 0.026 in South America). ${ }^{13}$ Based on the literature review, the cumulative probability of death by 12 months ranged from 0.03 to 0.29 , depending on the baseline CD4 cell count in the various populations that initiated ART. The literature review also found that mortality varied substantially by geographical region and by average starting CD4 cell level. ${ }^{13}$

Survival time in the years following the first year on treatment was also calculated. Using data from ART-LINC the probability of death between month 12 and month 24 was estimated to be 0.018 . It was estimated at 0.013 between month 24 and month 36 and 0.01 between months 36 and 48 . However, the literature review found higher estimates of mortality in the years after the first year on treatment. The estimated probability of death between month 12 and month 24 was 0.05 based on nine studies. Due to the small amount of evidence for survival in the years after 24 months since start on treatment, the same probability of death, 0.02 , was used for all subsequent years.

\section{Loss to follow-up on ART}

The literature review of survival on ART also collated data on loss to follow-up 12 months after ART initiation. Based on existing studies in the literature review (56 reports with appropriate data) between $1 \%$ and $15 \%$ of patients were lost to follow-up (this excludes one study in which $59 \%$ of patients were lost to follow-up). ${ }^{13}$ Calculating the mean from the studies we estimate that $10.2 \%$ of individuals who started ART were lost to follow-up at 12 months in lower-income countries (weighted by sample size).

According to the 2008 ART-LINC data $20 \%$ of patients who started ART were lost to follow-up after 12 months. ${ }^{13}$ The probability of loss to follow-up varied by region: 0.201 in Africa, 0.274 in Asia and 0.102 in South America.

Rosen and colleagues ${ }^{17}$ conducted a systematic review of patient retention in ART programmes in sub-Saharan Africa, which estimated retention rates (including mortality, loss to follow-up and discontinuing treatment) of $75.1 \%$ at 12 months. The study looked specifically at non-research programmes. The authors do not provide estimates of loss to follow-up alone; however, they do estimate that mortality was on average $4.2 \%$, making the estimated loss to follow-up rate somewhere just below $20.7 \%$ (assuming that the percentage discontinuing treatment but remaining in care is very small).

A simple average of the above three statistics provided a value of $17 \%$ patients lost to follow-up at 12 months. This value was applied as an estimate for loss to follow-up for national ART programmes in the first year of ART treatment.

This rate was similar to retention rates at 12 months reported through national programmes of $79.5 \%$ globally and $75.2 \%$ in sub-Saharan Africa. ${ }^{18}$ If mortality on ART is approximately $6 \%$ in the first year of life, and loss to follow-up on ART is $17 \%$, this would suggest an approximate $77 \%$ retention rate after 12 months (again, assuming the percentage discontinuing treatment but remaining in care is small).

The ART-LINC data also provided estimates of loss to followup for up to 4 years after initiation of treatment. Based on the increasing numbers of individuals lost to follow-up each year in the eART-linc data, we assumed the attrition rate would remain constant in subsequent years.

\section{Survival of those lost to follow-up}

If those lost to follow-up have higher mortality rates than those still in the ART programme the models based on right-censored data would underestimate mortality rates in persons living with HIV. To estimate mortality among those individuals who have been lost to follow-up, a review was conducted of available literature by Brinkhof and colleagues. ${ }^{19}$ The authors searched 
PubMed, EMBASE, Latin American and Caribbean Health Sciences Literature (LILACS), Indian Medlars Centre (IndMed) and African Index Medicus databases and the abstracts of three conferences for studies that traced patients lost to follow-up to ascertain their vital status. Key variables considered in the analysis included the proportion of patients traced, the proportion found to be alive and the proportion that had died. Mortality data from several studies were combined using random-effects meta-analysis. Seventeen studies were eligible. All were from sub-Saharan Africa, except one study from India. A total of 6420 patients (range 44-1343 patients) was included. Patients were traced using telephone calls, home visits and through social networks. Overall, the vital status of 4021 patients could be ascertained $(63 \%$, range across studies $45-86 \%)^{19}$

Among the 4021 patients lost to follow-up 1602 patients had died ( $40 \%$, range $12-87 \%)$. The combined mortality estimate was $40 \%$ (95\% CI 33\% to 48\%), with substantial between-study heterogeneity $(p<0.0001)$. When restricting the analysis to patients starting ART in public programmes in Africa (12 studies), combined mortality was $47 \%$ (95\% CI 39\% to $54 \%$, p for heterogeneity <0.0001). The authors of that review concluded that in ART programmes in resource-limited settings among those lost to follow-up typically $30-60 \%$ had died. The proportion of patients classified as lost to follow-up who were in fact patients who had transferred to continue ART in another ART site ranged from $12 \%$ to $54 \%$ in the studies reviewed. The estimate restricted to the public programmes (47\%) was determined to be the value most appropriate for the national programmes using Spectrum.

The estimate of $47 \%$ mortality can be assumed to be in the first year. The study was not able to provide the mortality levels over different time periods so the same value was used for subsequent years.

Based on the above information the parameters described in table 1 were used in the Spectrum model to describe the survival of those adults who start ART programmes.

\section{CHILDREN}

In July 2008, Unicef, UNAIDS and WHO sponsored a consultation to review the parameters related to children in Spectrum. Two studies were commissioned for this consultation to determine child HIV progression parameters for Spectrum. ${ }^{20}$

A description of the derivation of the parameters related to time from overall seroconversion to death for children is provided elsewhere. ${ }^{2}$ In brief, a recent pooled analysis used results from eight study sites to create separate survival schedules for children infected perinatally and those infected through breastfeeding. ${ }^{21}$ Progression from HIV seroconversion to death was revised to follow these schedules in Spectrum.

\section{Seroconversion until need for treatment}

In the 2009 version of Spectrum eligibility criteria for children are now adjustable to match the country's treatment strategy. The default settings in Spectrum reflect the timing and criteria of the WHO guidelines as they have been implemented. From 2000 until 2006 eligibility for ART among children was based on a graduated CD4 cell count or percentage depending on age (see table 2). From 2007 to 2009 the guidelines stated that all children identified as HIV positive under 12 months were eligible. In 2010 WHO published revised guidelines recommending that all HIVpositive children under 24 months be provided with ART and children aged 24-59 months were eligible for ART if their CD4
Table 1 Calculation of adult survival parameters used in Spectrum

\begin{tabular}{ll}
\hline Survival in first year & \\
Mortality rate in first year of treatment & 0.06 \\
Proportion lost to follow-up & 0.17 \\
Mortality rate of those lost to follow-up & 0.47 \\
Survival at end of first year & $1-\left(0.06+\left(0.17^{*} 0.47\right)\right)=0.86$ \\
Survival in subsequent years & \\
Mortality rate in subsequent years & 0.02 \\
Proportion lost to follow-up & 0.17 \\
Mortality rate of those lost to follow-up & 0.47 \\
Survival in subsequent years & $1-\left(0.02+\left(0.17^{*} 0.47\right)\right)=0.90$ \\
\hline
\end{tabular}

cell percentage was less than $25 \%$ or CD4 cell count was less than 750 cells $/ \mathrm{mm}^{3}{ }^{22}$

Spectrum estimates the proportion of HIV-infected children eligible for treatment by using patterns of the distribution of HIV-infected children by CD4 cell count or CD4 cell percentage. These patterns were derived from the HIV Paediatric Prognostic Markers Collaborative Study (HPPMCS), which took place in western countries before ART was available. This is a unique dataset because it includes data on CD4 cell percentage and count in a setting in which no ART is available. Such data are not available from low and middle-income countries. The study included a meta-analysis of individual longitudinal data on 3941 HIV-1-infected children enrolled in cohort studies and randomised control trials in the USA and Europe. Care was taken to avoid using the same children in multiple studies. ${ }^{23}$

The distribution of CD4 cell count and percentage CD4 cells from HPPMCS provide a basis by which to progress children from birth to the need for treatment in the Spectrum model. All HIV-infected children less than 1 year old are assigned the CD4 cell distributions from the HPPMCS. The patterns in figure 2 were used to calculate the proportion of HIV-infected children at each age and CD4 cell category that remain in the same CD4 cell category the following year or drop down to a lower CD4 cell category. Spectrum thus reproduces these patterns in the absence of ART and the number of children eligible for ART by age can be determined based on the country-specific eligibility criteria. As ART coverage increases children are drawn from the eligible categories leaving an altered distribution of HIV-infected children who are not on ART.

\section{Survival on ART}

In 2008, Dabis and colleagues conducted a literature review on child survival on ART in lower-income countries on behalf of

Table 2 Default ART eligibility criteria for children in Spectrum

\begin{tabular}{lccc}
\hline & $\mathbf{2 0 0 0 - 6}$ & $\mathbf{2 0 0 7 - 9}$ & $\mathbf{2 0 1 0 - 1 5}$ \\
\hline $\begin{array}{l}\text { Age below which all HIV-positive children } \\
\text { receive treatment (months) }\end{array}$ & 0 & 12 & 24 \\
CD4 cell count threshold for eligibility & & & \\
Age $<11$ months & 1500 & 1500 & 750 \\
Age $12-35$ months & 750 & 750 & 750 \\
Age $35-59$ months & 350 & 350 & 750 \\
Age $\geq 5$ years & 200 & 200 & 350 \\
CD4 cell percentage threshold for eligibility & & & \\
Age $<11$ months & 25 & 25 & 25 \\
Age $12-35$ months & 25 & 25 & 25 \\
Age $35-59$ months & 25 & 25 & 25 \\
Age $\geq 5$ years & 15 & 15 & 15 \\
\hline
\end{tabular}

$\mathrm{ART}$, antiretroviral therapy. 

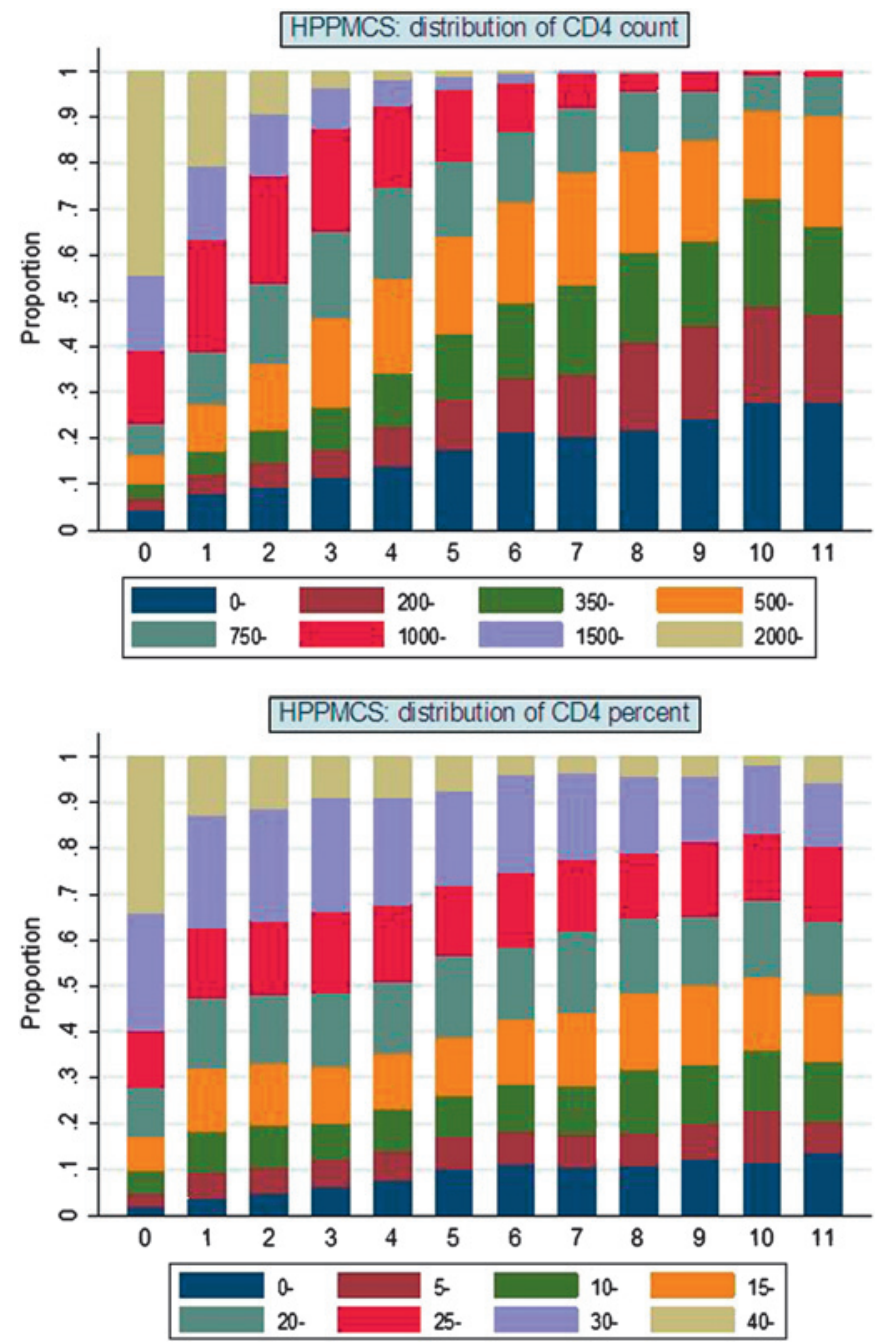

Figure 2 Distribution of CD4 cell count and CD4 cell percentage by time since seroconversion (year), HIV Paediatric Prognostic Markers Collaborative Study (HPPMCS).

Unicef. Articles were selected after screening of PubMed/ Medline and Scopus up to March 2008 and abstracts of 2007-8 international conferences. Observational cohorts, clinical trials and programme reports were eligible as long as they took place in lower-income countries and included children on ART or on ART and cotrimoxazole. Only studies with death as the outcome were included in this paediatric ART review update. The extracted data included patient's demographic characteristics, baseline CD4 cell count, survival estimates and factors associated with mortality of children receiving cotrimoxazole and/or ART. A total of 14 prospective studies was eligible for the analysis. ${ }^{20}$

The amount of data on the survival of HIV-infected children on ART in lower and middle income countries more than doubled since a previous review that covered a period ending in December $2006 .^{12}$ Sample sizes were generally larger, exceeding 400 participants for some of the studies, thereby increasing the accuracy of the findings. Some reports allowed for the production of survival estimates up to 24 months after ART initiation.

The authors concluded that the probability that infants survive the first year of treatment was estimated to be 0.93-0.95 and survival until 2 years was estimated to be 0.91-0.92. The authors estimated that the provision of cotrimoxazole along with ART additionally reduced mortality by approximately $33 \%$ in the first year, $16 \%$ in the second year and $8 \%$ in the third year. ${ }^{20}$ Spectrum allows the user to specify the proportion of HIV-exposed children receiving cotrimoxazole and adjusts mortality accordingly.

\section{Loss to follow-up on ART}

The above study also compiled available data on loss to followup among children. Very few data were available at the time of finalising the Spectrum model, resulting in the use of expert opinion to estimate the parameter. The model assumes that an estimated $10 \%$ of children were lost to follow-up and among those, $50 \%$ were expected to have died within 1 year.

Taking into account the additional mortality among those lost to follow-up, the estimated survival among those children who started on ART was 0.89 in the first year on ART and 0.93 for subsequent years. To compensate for the increased survival due to cotrimoxazole, a value of 0.85 was used to describe survival in the first year among children starting ART.

\section{LIMITATIONS}

The parameters were derived from the analysis of available databases and the results published in peer-reviewed scientific literature. However, there are a number of limitations due to the lack of consistency in outcome measures between published studies and the lack of data available from which to estimate a robust parameter.

Most of the published articles present survival and retention for 12 months while only a few studies provide information for longer durations. Parameters for longer durations will improve the accuracy of the models.

There could be some overestimation to the loss to follow-up rates estimated from the ART-LINC of IeDEA data. This database did not systematically record when patients were transferred to another centre for follow-up. This information may have been taken into account in some of the other published studies reducing the comparability of the data. In addition, loss to follow-up is computed differently depending on the study site, again reducing the comparability of the data.

The ART-LINC database was composed of 19 cohorts and two networks and until 2008 constituted the largest available dataset in lower-income countries. Some data from ART-LINC may originate from the same clinics as the data used in the individual cohorts that published articles. It is often difficult to identify which part of the ART-LINC dataset overlaps with a published report so all data were used. Also in the systematic review of published data articles reporting on the same study were included if the population study was not exactly the same or the outcomes differed.

The ART-LINC data are very useful for computing such parameters as they are organised in a single dataset of individuallevel patient data. However, these data do not always reflect the situation at the national level. Many of the sites included in the ART-LINC data are research or private clinics and are not representative of the average experience in a public clinic.

In addition the ART-LINC dataset is not large enough to represent all of the regions where Spectrum is used. Countries in Europe and Latin America often find that the default parameters in Spectrum do not reflect the situation in their countries. In such cases, countries are encouraged to collect data that might inform the parameters from their own country. The creation of regional data centres by the IeDEA initiative of the US National Institutes of Health in 2007 (http://www.iedea-hiv.org/) will ultimately lead to regional and possibly country-specific estimates in the future. 
The review of survival time on ART showed that this parameter varied greatly by average CD4 cell count at the start of treatment. This was also evident in the ART-LINC estimates of probability of death. Future versions of Spectrum will incorporate an option that allows countries to describe the distribution of CD4 cell counts at the start of treatment to create a more accurate estimated duration of survival on ART. In addition, loss to -follow-up varies by CD4 cell count at the start of treatment according to the ART-LINC data, again suggesting the need to create a model that varies the retention on ART parameter estimate based on average starting CD4 cell values. If countries are able to populate the models with the distribution of starting CD4 cell counts the need for region-specific parameters will be reduced.

Finally, the data on HIV progression from the HPPMCS study among children in the USA and western Europe could potentially be different than in low and middle-income country settings where children have different nutrition levels and underlying health.

\section{CONCLUSIONS}

The UNAIDS HIV estimates published in 2010 are based on recent evidence. Data describing survival on ART for adults and children were used to refine the estimate of survival in the first year of ART and the subsequent years for adults and children. Ideally, countries will use their own country-specific data on ART survival, loss to follow-up and survival among those lost to follow-up in Spectrum to create more accurate estimates of the impact of HIV on their populations. However, when those data are not available default parameters are included in the programme (see table 3 ).

Since the use of Spectrum by countries to create national estimates, additional data have become available to inform the validity of these parameters. More recent studies show that mortality among adults lost to follow-up actually decreases in subsequent years. ${ }^{24-26}$ In future models the parameters will indicate lower mortality in patients lost to follow-up after the first year on ART. A recently conducted analysis of loss to follow-up data of children on ART indicated that the percentage lost to follow-up at 18 months was $12.3 \%$ among 13611 children on ART in the context of the IeDEA initiative. Analysing these data by region found significant variation by region: $4.1 \%$ in Asia; $14.0 \%$ in east Africa; $9.0 \%$ in southern Africa and $21.8 \%$ in west Africa $(p<0.0001){ }^{27}$

To improve the accuracy of estimates, countries should compare mortality among those lost to follow-up to death registries where available. Two recent studies from South Africa were able to correct mortality estimates and more accurately estimate programme effectiveness by linking results to vital registration information. ${ }^{16} 18$

Although countries are encouraged to monitor retention rates in ART programmes routinely, ${ }^{28}$ those data can not be used directly in the Spectrum model at this point. Spectrum requires information on the survival of those persons in the ART

Table 3 Survival parameters and their values

\begin{tabular}{lll}
\hline Parameter & Value & Sources \\
\hline Adults & & \\
$\quad \begin{array}{l}\text { Survival in first year } \\
\quad \text { Survival in subsequent years }\end{array}$ & 0.86 & Lewden et $a l^{13}$ and Brinkhof et $a l^{19}$ \\
Children & & Lewden et $a l^{13}$ and Brinkhof et $a l^{19}$ \\
$\quad \begin{array}{l}\text { Survival in first year } \\
\quad \text { Survival in subsequent years }\end{array}$ & 0.95 & UNAIDS et al ${ }^{20}$ \\
\end{tabular}

\section{Key messages}

UNAIDS HIV estimates published in 2010 are based on recent data on survival on ART for adults and children.

- Data from adult cohort studies were used to estimate progression from infection to eligibility for treatment under three different CD4 cell count criteria: less than 200, less than 250 and less than 350 cells $/ \mathrm{mm}^{3}$.

- Spectrum now has the flexibility to define children eligible for treatment on the basis of age, CD4 cell count and CD4 cell percentage.

programme as well as those lost to follow-up in order to progress each subpopulation accurately.

The default parameters used in Spectrum are calculated for low and middle-income countries. These parameters might not be appropriate for models of epidemics in high-income countries. HIV estimates for high-income countries are currently still derived using Spectrum due to a lack of alternative models.

The Spectrum parameters and functions related to time from seroconversion to treatment eligibility and survival on ART have been regularly updated based on available data and model needs. The new capability to adjust both child and adult eligibility criteria will make the model more appropriate for the existing situations in countries. Additional recent data on survival on ART for adults and children were incorporated to refine the estimate of survival in the first year of ART and the subsequent years for adults and children. The addition of information on loss to follow-up and survival among those lost to follow-up will improve the estimates of AIDS-related mortality as well as those in need of treatment.

Every 2 years the parameters in Spectrum will continue to be revised as additional data become available. This process has improved the accuracy and precision of HIV estimates and projections over the past 10 years.

Acknowledgements The authors would like to thank the HIV Paediatric Prognostic Markers Collaborative Study for providing data and figures on their results.

\section{Competing interests None declared.}

Contributors MM conceptualised and drafted the manuscript; CL led the research on adult survival on antiretroviral therapy and loss to follow-up; MWGB led the research on survival among adults lost to follow-up; FD led the research on the survival parameters for children; JMT provided comments on the manuscript; YS provided comments on the manuscript; JS developed the Spectrum package and drafted parts of manuscript.

Provenance and peer review Not commissioned; externally peer reviewed.

\section{REFERENCES}

1. Brown T, Bao L, Raftery AE, et al. Modelling HIV epidemics in the antiretroviral era: the UNAIDS estimation and projection package 2009. Sex Transm Infect Supplement 2010;86(Suppl II):ii3-ii10.

2. Stover J, Johnson $P$, Hallett $T$, et al. The Spectrum projection package: improvements in estimating incidence by age and sex, mother-to-child transmission, HIV progression in children and double orphans. Sex Transm Infect 2010;86(Suppl II): ii16-ii21.

3. Duncan J, Beckford Jarrett S, Harvey K. Using estimation and projection package and Spectrum for Jamaica's national HIV estimates and targets. Sex Transm Infect 2010;86(Suppl II):ii43-ii47.

4. Stanecki K, Daher J, Stover J, et al. Under-5 mortality due to HIV: regional levels and 1990-2009 trends. Sex Transm Infect 2010;86(Suppl II):ii56-ii61.

5. Mahy M, Stover J, Stanecki K, et al. Estimating the impact of antiretroviral therapy: regional and global estimates of life-years gained among adults. Sex Transm Infect 2010;86(Suppl II):ii67-ii71.

6. Stover J. Aim: a computer program for making HIVIAIDS projectinos and examining the demographic and social impacts of AIDS. Glastonbury, CT: Futures Institute, 
2009. http://data.unaids.org/pub/Manual/2009/20090414 aim manual 2009 en. pdf (accessed 15 Sep 2010).

7. Stover J, Johnson P, Zaba B, et al. The Spectrum projection package: improvements in estimating mortality, ART needs, PMTCT impact and uncertainty bounds. Sex Transm Infect 2008;84(Suppl 1):i24-30.

8. Todd J, Glynn J, Marston M, et al. Time from HIV seroconversion to death: a collaborative analysis of eight studies in six low and middle-income countries before highly active antiretroviral therapy. AIDS 2007;21(Suppl 6):S55-63.

9. Ghys PD, Zaba B. Prins M. Survival and mortality of people infected with HIV in low and middle income countries: results from the extended ALPHA network. AIDS 2007;21(Suppl 6):S1-4.

10. eART-Linc. Duration from seroconversion to eligibility for antiretroviral therapy and from ART eligibility to death in adult HIV-infected patients from low and middleincome countries: collaborative analysis of prospective studies. Sex Transm Infect 2008;84(Suppl 1):i31-6.

11. Braitstein P, Brinkhof MW, Dabis F. Mortality of HIV-1-infected patients in the first year of antiretroviral therapy: comparison between low-income and high-income countries. Lancet 2006;367:817-24.

12. Coffie $\mathbf{P}$, Moh R, Ekouevi DK, et al. Survival of HIV-infected adults and children on antiretroviral therapy in low and middle-incomes countries. TECHNICAL Report for UNAIDS. Bordeaux: Institut de Santé Publique, Epidémiologie et Développement (ISPED) Université Victor Segalen, 2007

13. Lewden C, Balestre E, Dabis F. Survival and loss-to-follow-up of HIV-infected adults who have started antiretroviral therapy in low and middle-income countries. Technical Report for UNAIDS. Bordeaux: Institut de Santé Publique, d'Epidémiologie et de Développement (ISPED) Université Victor Segalen, 2009.

14. Severe $\mathbf{P}$, Pape J, Fitzgerald D. A randomized clinical trial of early versus standard antiretroviral therapy for HIV-infected patients with a CD4 T cell count of 200-350 cells/ml (CIPRAHT001). 49th Interscience Conference on Antimicrobial Agents and Chemotherapy. San Francisco, CA, 15-19 September 2009. Abstract H-1230c.

15. Emery S, Neuhaus J. Phillips A et al. Major clinical outcomes in antiretroviral therapy (ART) - naive participants and in those not receiving ART at baseline in the SMART Study. J Infect Dis 2008;197:1133-44.

16. World Health Organization. Antiretroviral therapy for HIV infection in adults and adolescents. Geneva: World Health Organization, 2010. http://whqlibdoc.who.int/ publications/2010/9789241599764 eng.pdf (accessed 16 Aug 2010).

17. Rosen S, Fox MP, Gill CJ. Patient retention in antiretroviral therapy programs in subsaharan africa: a systematic review. PLoS Med 2007;4:e298.
18. Tassie J-M, Baijal P, Vitoria MA, et al. Trends in retention on antiretroviral therapy in national programs in low-income and middle-income countries. J Acquir Immune Defic Syndr 2010;54:437-41.

19. Brinkhof MWG, Pujades-Rodriguez M, Egger M. Mortality of patients lost to followup in antiretroviral treatment programmes in resource-limited settings: systematic review and meta-analysis. PLoS One 2009;4:e5790.

20. UNAIDS, World Health Organization, UNICEF. Consultative meeting on data collection and estimation methods related to HIV infection in infants and children. New York: UNICEF, 2008. http://www.epidem.org/Publications/UNAIDS UNICEF Paediatric\%20HIV\%20Report 9\%20Dec\%202008 Final.pdf laccessed 18 Mar 2010)

21. Becquet R; UNAIDS Child Survival Working Group. Survival of children HIV-infected perinatally or through breastfeeding: a pooled analysis of individual data from subSaharan Africa. 17th Conference on Retrovirals and Opportunistic Infections. San Francisco, CA, 16-19 February 2010. Paper 840.

22. Wold Health Organization. Antiretroviral therapy of HIV infection in infants and children: recommendations for a public health approach. Geneva: World Health Organization, 2010. http://www.who.int/hiv/pub/guidelines/paediatric020907.pdf (accessed 16 Aug 2010)

23. Dunn D. Short-term risk of disease progression in HIV-1-infected children receiving no antiretroviral therapy or zidovudine monotherapy: a meta-analysis. Lancet 2003;362:1605-11.

24. Fox MP, Brennan A, Maskew M, et al. Using vital registration data to update mortality among patients lost to follow-up from ART programmes: evidence from the Themba Lethu Clinic, South Africa. Trop Med Int Health 2010:15:405-13

25. Geng EH, Bangsberg DR, Musinguzi N, et al. Understanding reasons for and outcomes of patients lost to follow-up in antiretroviral therapy programs in Africa through a sampling-based approach. J Acquir Immune Defic Syndr 2010;53:405-11

26. Boulle A, Van Cutsem G, Hilderbrand K, et al. Seven-year experience of a primary care antiretroviral treatment programme in Khayelitsha, South Africa. AIDS 2010:24:563-72

27. Leroy V, Malateste K, Rabie H. 18-Month mortality and loss to follow-up in ARTtreated children in Asia and Africa. XVIIIth International AIDS Conference, Vienna, Austria, 18-23 July, 2010.

28. UNAIDS. Monitoring the Declaration of Commitment on HIV/AIDS: Guidelines on Construction of Core Indicators - 2010 Reporting. Geneva: UNAIDS, 2009 